This work was supported in part by U.S. Public Health Service research grant GM 10177 from the National Institutes of Health.

Physies Department,

University of Tasmania,

Hobart, Tasmania.

${ }^{1}$ Canny, M. J., Biol. Rev., 35 (4), 507 (1960).

${ }^{2}$ Goldsmith, M. H. M., and Thimann, K. V., Plant Physiol., 37 (4), 492 (1962).

${ }^{3}$ Newman, I. A., Nature, 184, 1728 (1959).

${ }^{4}$ Newman, I. A., Austral. J. Biol. Sci., 16 (3), 629 (1963),

\section{In vitro Production of Neomorphs in Anethum graveolens L.}

Is an earlier communication Johri and Sehgal' ${ }^{1}$ reported the chemical induction of polyembryony in Anethum graveolens. The work recorded here is an extension of that investigation.

In Nature the fruits mature approximately 25 days after pollination and the seeds show a copious fatty endosperm and a single minute embryo.

Young ovaries, three days after pollination (Fig. 1), were grown under aseptic conditions on a modified White's medium containing 4 per cent sucrose. This would be referred to as the basal medium. Casein hydrolysate, yeast extract and indolyl-3-acetic acid were used as supplements.

At the time of inoculation the ovules showed the zygote and a free nuclear endosperm. The ovaries implanted on basal medium and basal medium + indolyl-3-acetic acid
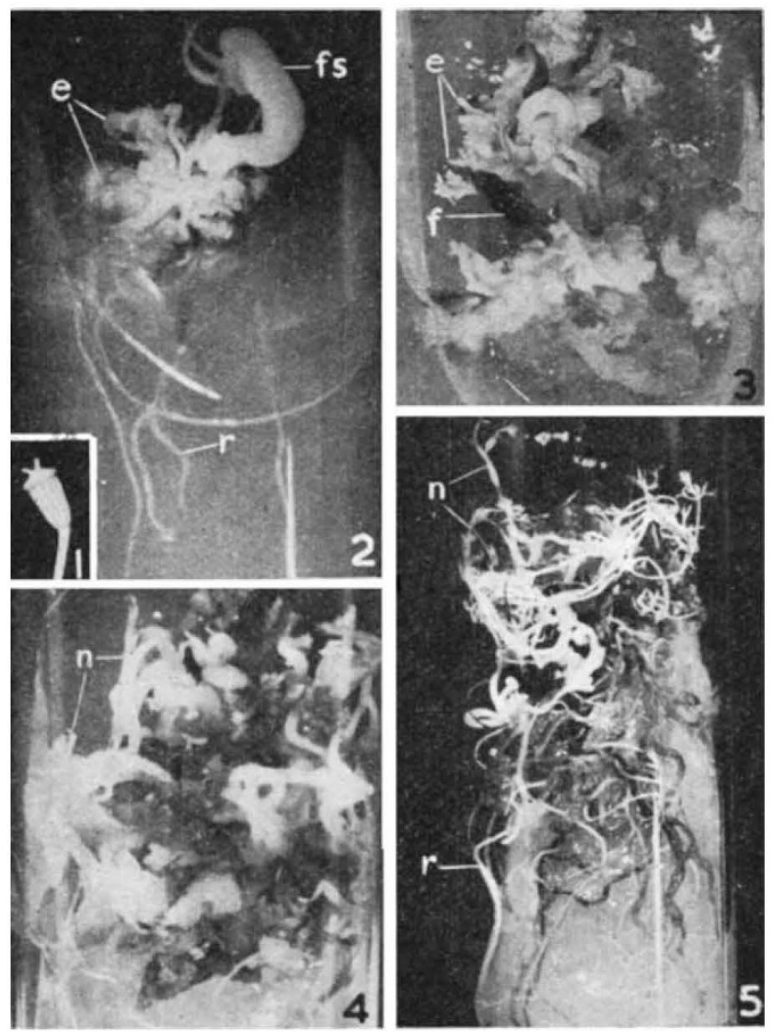

Fig. 1. Ovary (stage of inoculation) 3 days after pollination $(\times c .2 \cdot 6)$. Fig. 2. 32-week-old culture on basal medium + yeast extract $(1,000$ p.p.m.) with a fasciated seedling and several undifferentiated embryo-
like structures $(x c, 1 \cdot 66)$

Fig. $3(\times c .1 \cdot 7)$ and Fig. $4(\times c .1 \cdot 5)$. 43- and 46-week-old cultures on basal medium + casein hydrolysate $(i, 000$ p.p.m. $)+$ indolyl-3-acetic acid (1 p.p.m.) and basal medium + casein hydrolysate (1,000 p.p.m.) with numerous thalloid neomorphs

Fig. 5. 40-week-old culture on basal medium + casein hydrolysate (500 p.p.m.) showing coralloid neomorphs $(\times c .1 \cdot 7)$

$e$, Embryo-like structures; $f$, fruit; $f s$, fasciated seedling; $n$, neo$f$, fruit; $f s$, fast
morphs; $r$, root
(1, 5, 10 p.p.m.) produced only monoembryonate seeds, while basal medium + casein hydrolysate $(100,1,000$ p.p.m.) and basal medium + yeast extract $(100,1,000$ p.p.m.) -with or without the addition of indolyl-3-acetic acid (1 p.p.m.)-induced polyembryony in 6-20 per cent cultured ovaries. In 13- to 14-week-old cultures tho seeds germinated in situ and gave rise to normal seedlings. However, in a few cultures grown on basal medium + casein hydrolysate or basal medium + yeast extract, with or without indolyl-3-acetic acid, the seodlings were chlorotic and fasciated. Eight to 12 months later (cultures transferred through 4-6 passages of 8 weeks each) the fasciated tissues differentiated into embryo-like structures (Figs. 2 and 3 ) which finally developed into 1-2-cm-long thalloid (Fig. 4) or coralloid (Fig. 5) seedlings. These vegetatively reproducing plantlets, termed neomorphs, resemble the cotyledonary stage of a normal seedling. Further proliferation and multiplication of the neomorphs occurred in cultures maintained for 10-12 months (Figs. 3-5). On sub-culturing in basal medium, basal medium + casein hydrolysate or basal medium + yeast extract (with or without indolyl-3-acetic acid) for 6-8 weeks, they produced normal seedlings.

Earlier, Waris ${ }^{2,3}$ reported that the mature seeds of umbellifers like Cicuta virosa, Daucus carota, Oenanthe equatica and $O$. lachenalii grown on a liquid medium (for detailed composition of this medium see ref. 3) containing 1 per cent sucrose and $0 \cdot 1-0 \cdot 4$ per cent glycine produced more or less similar neomorphs. Miettinen and Waris ${ }^{4}$ noted that the neomorphs contained a low concentration of sugars but had free amino-acids in a 10-times higher concentration than normal individuals. They therefore concluded that the accumulation of soluble amino-acids in the neomorphs indicated a disturbance in protein synthesis. They further suggested that the neomorphic condition of seedlings is a physiological adaptation to a higher concentration of glycine and does not involve any irreversible change of genotype.

It is possible that in Anethum graveolens also the aminoacids present in casein hydrolysate and yeast extract accumulate in the seedlings which produce the neomorphs, but this has to be confirmed by chemical analysis. In any case, like the induction of zygotic polyembryony, the production of neomorphs can be exploited by plant breeders for raising a large population of seedlings with desired characters.

We are grateful to Prof. P. Maheshwari for his keen interest in the progress of this work.

One of us (C. B. S.) acknowledges receipt of a senior research fellowship from the Council of Scientific and Industrial Research, New Delhi.

B. M. JOHRI

C. B. SFHGAI

Department of Botany,

University of Delhi.

${ }^{1}$ Johri, B. M., and Sehgal, C. B., Naturwiss., 50, 47 (1963).

${ }^{2}$ Waris, H., Physiol. Plant., 12, 753 (1959).

${ }^{3}$ Waris, H., Physiol. Plant., 15, 736 (1962).

${ }^{4}$ Miettinen, Jorma K., and Waris, H., Physiol. Plant.,11, 193 (1958).

\section{Giant Chromocentres and Nucleoli in Lumbricus}

FURTHER observations on intranuclear aspects of giant nuclei found in the pharyngeal epithelium in Lumbricus have been made. A previous report was given concerning these nuclei ${ }^{1}$. Not only were the overall dimensions of these nuclei enlarged when compared with the more typical nuclei, but also on examination of the intranuclear mor phology it became apparent that giant chromocentres (Figs. 1, 4 and 6), giant nucleoli (Figs. 1, 2, 3, 4, 5 and 6), and even giant nucleolar organizers (Figs. 3 and 4) were present.

Endopolyploidy could explain these features if it were assumed that not only nucleolar fusion exists but also 